\title{
Early Stress Is Associated with Alterations in the Orbitofrontal Cortex: A Tensor-Based Morphometry Investigation of Brain Structure and Behavioral Risk
}

\author{
Jamie L. Hanson, ${ }^{1,2}$ Moo K. Chung, ${ }^{1}$ Brian B. Avants, ${ }^{3}$ Elizabeth A. Shirtcliff, ${ }^{1}$ James C. Gee, ${ }^{3}$ Richard J. Davidson, ${ }^{1,2}$ \\ and Seth D. Pollak ${ }^{1,2}$ \\ ${ }^{1}$ Waisman Center, University of Wisconsin-Madison, Madison, Wisconsin 53705, 22Department of Psychology, University of Wisconsin-Madison, Madison, \\ Wisconsin 53703, and ${ }^{3}$ Penn Image Computing and Science Laboratory, Department of Radiology, University of Pennsylvania, Philadelphia, Pennsylvania \\ 19104
}

Individuals who experience early adversity, such as child maltreatment, are at heightened risk for a broad array of social and health difficulties. However, little is known about how this behavioral risk is instantiated in the brain. Here we examine a neurobiological contribution to individual differences in human behavior using methodology appropriate for use with pediatric populations paired with an in-depth measure of social behavior. We show that alterations in the orbitofrontal cortex among individuals who experienced physical abuse are related to social difficulties. These data suggest a biological mechanism linking early social learning to later behavioral outcomes.

\section{Introduction}

Individuals who suffer early adversity, such as child maltreatment, are at heightened risk for a variety of behavioral, social, and health problems throughout their lives (Cicchetti and Carlson, 1989). This occurrence of early stress raises complex issues about human developmental biology and represents the classic problem of how early experiences influence the nature and trajectory of brain development. Here we examine the effects of early adverse parental care on a brain system responsible for regulation of social behavior in human children.

Little is understood about the neurobiological mechanisms underlying the broad range of difficulties observed among individuals who experienced early child abuse (Gilbert et al., 2009). We focused our examination on development of the orbitofrontal cortex (oFC) in these children because this brain region is central to emotion and social regulation (Damasio, 1994; Rempel-Clower, 2007) and when disrupted, confers an increased vulnerability for the development of mental health problems such as depression or substance abuse (Paulus, 2007). Functional neuroimaging studies point to this orbital sector of the prefrontal

Received Feb. 16, 2010; revised March 22, 2010; accepted March 26, 2010.

This work was supported by National Institutes of Health Research Grants MH61285 and MH68858 to S.D.P. funded by the National Institute of Mental Health and the Children's Bureau of the Administration on Children, Youth and Families as part of the Child Neglect Research Consortium, as well as National Institute of Mental Health Grant MH84051 to R.J.D. We thank Andrew Alexander, Michael Anderle, Lisa Angelos, Patrick Bauer, Aaron Cohn, Johnna Dorshorst, Erin Eatough, Ronald Fisher, and Chastity Jensen for help with data collection and Andrew Fox, Brendon Nacewicz, Terrence Oakes, and Nicole Strang for helpful discussions.

Correspondence should be addressed to Jamie L. Hanson, Waisman Center and Department of Psychology, University of Wisconsin-Madison, 1500 Highland Avenue, Madison, WI 53705. E-mail: jlhanson5@wisc.edu.

E. A. Shirtcliff's current address: Department of Psychology, University of New Orleans, Geology/Psychology Building, Lakefront Campus, 2000 Lakeshore Drive, New Orleans, LA 70148

DOI:10.1523/JNEUROSCI.0859-10.2010

Copyright $\odot 2010$ the authors $\quad 0270-6474 / 10 / 307466-07 \$ 15.00 / 0$ cortex (PFC) as supporting behaviors such as inhibition, appropriate responses to other people's moods, and self-regulation of social-emotional behavior (Bachevalier and Loveland, 2006).

Longitudinal neuroimaging studies suggest that the oFC is one of the last regions in the brain to fully develop in humans. The oFC may therefore be particularly vulnerable to the effects of early stress, as changes in the oFC are seen until 20 years of age or later (Toga et al., 2006). Nonhuman animal models suggest that early stress is associated with alterations in portions of the PFC (Braun et al., 2000). As an example, infant rodents had lower spine densities and shorter apical dendrites in the orbitofrontal cortex when separated from one of their parents (Helmeke et al., 2009). Similar alterations in the PFC have also been noted in rhesus macaques, separated in infancy from their mothers at 2 months of age, with decreases noted in white matter in the PFC compared with control-comparison monkeys (Sánchez et al., 1998).

We assessed the relationship between development of the oFC and the social functioning of children who were verified victims of physical abuse by using a tensor-based morphometry (TBM) framework (Chung et al., 2001). TBM uses the Jacobian determinant of the deformation field that is required to register one brain to another to detect volumetric changes. We also evaluated and controlled for pubertal stage, which may be affected by early adversity (Ellis and Garber, 2000), thereby influencing brain development (Peper et al., 2009).

We hypothesized that the orbitofrontal cortex would be smaller in children who had experienced prolonged maltreatment and, since this region is important for behavioral and emotional flexibility, these alterations would be predictive of children's adaptive social behavior. To test this, we assessed each child's competence in stressregulation across numerous social domains, both indexing the ob- 
jective impact of stressful events as well as the child's subjective experience of the event.

\section{Materials and Methods}

Participants. Seventy-two children (31 physically abused, 41 nonabused) participated in this study. Physically abused children and their families were recruited by letters forwarded by the Department of Human Services in Dane County, WI, to families with substantiated cases of physical maltreatment. Nonabused children were recruited from the Madison community by posting fliers. Parents completed the Parent-Child Conflict Tactics Scale (PC-CTS) (Straus et al., 1998), a 20-item measure of the frequency with which a parent has performed specific acts of physical aggression toward the child. To qualify as controls in the present study, children were required to have scores $<12$ on the PC-CTS. Children whose parents scored at least 20 on the physical abuse subscale on the PC-CTS and/or had substantiated cases of physical abuse on record with the Dane County Department of Human Services were classified as abused.

Groups were similar on demographic variables (for additional information, see supplemental Materials, available at www.jneurosci.org). Physically abused subjects had smaller overall whole-brain volumes (physically abused: $1387.57 \pm 134.26 \mathrm{~cm}^{3}$; typically developing: $1466.98 \pm 121.09$ $\left.\mathrm{cm}^{3} ; F=6.91 ; p=0.011\right)$. Subsequent analyses took this difference into account.

Pubertal examination. All children completed a physical examination with Tanner's staging (Marshall and Tanner, 1969, 1970). Experienced pediatric nurse practitioners conducted physical examinations for research purposes (Shirtcliff et al., 2009). The clinical examination for girls assessed breast development with brief palpation and visual examination of pubic hair. An orchidometer was used to measure testicular size in boys (Genentech, 1997), along with visual inspection of pubic hair. Interobserver reliability between the nurse practitioners $(N=10)$ was good, $\kappa=0.88$. Puberty was rated ranging from 1 (no development) to 5 (adult development) (Marshall and Tanner, 1970). There was a trend for abused children to have more advanced pubertal development (physically abused: $1.758 \pm 1.0791$; typically developing: $1.354 \pm 1.1401 ; F=$ $2.325, p=0.132)$.

Fetal alcohol exposure screening. To rule out prenatal alcohol exposure, a medical geneticist who specializes in fetal alcohol syndrome reviewed facial photographs of all participants. These photographs examined (1) distance between the endocanthion and exocanthion landmarks, (2) philtrum smoothness, and (3) upper lip thinness. This screening tool has demonstrated very high sensitivity and specificity for prenatal alcohol exposure (Astley et al., 2002). No children from either group had signs of fetal alcohol exposure or fetal alcohol syndrome.

Neuropsychological functioning. Children's cognitive functioning was assayed using the Cambridge Neuropsychological Test Automated Battery (CANTAB), a computerized series of neuropsychological tests used extensively with children and adolescents (Luciana and Nelson, 2002).

Assessment of secondary psychopathology. To assess child psychopathology, parents completed the computerized Diagnostic Inventory for Children and Adolescents (DICA-P) (Reich, 2000) regarding their child. Responses to this computerized program were used to generate specific Diagnostic and Statistical Manual of Mental Disorders (DSM) IV diagnoses.

Interview methods. To assess psychological functioning, parents and children each separately completed the Life-Stress Interview (LSI) (Rudolph and Hammen, 1999). This semi-structured interview assessed stressful life events of both chronic and episodic nature. Interviewers received intensive training from the developers (of the LSI) at the University of Illinois at Urbana-Champaign. Children were interviewed regarding stressors in domains relevant to their lives, e.g., school, peer, and family. Parents were also asked about their child's functioning within these various domains. Advanced graduate-level researchers conducted all interviews. A panel of 3-6 trained raters then scored the impact of each life event based on parent's and child's separate reports on a 5-point scale (1-5, from no or minimal impact to severe impact) using a scale developed by Rudolph and Hammen (1999). Increasing scores are related to more serious difficulties in each subdomain. For example, a score of 1 on the academic subscale is demonstrative of a child getting mostly
A's and doing above average work, while a score of 5 would be given with the failure of 3 or more subjects in 1 year and full-time placement in a learning disabilities class. Once parent and child reports were scored individually, a consensual report was made regarding the child by integrating information from both informants. The ratings of episodic stress between parent and child varied slightly across domain, but were significantly correlated, with $r$ values $>0.57$ and $p$ values $<0.001$ (LSIacademic $r=0.803, p<0.001$; LSI-behavioral $r=0.639, p<0.001$; LSI-peer $r=0.573, p<0.001$; LSI-family $r=0.698, p<0.001)$. High reliability has previously been achieved for the interviews classification of stressful life events (intraclass correlation coefficients of 0.85) (Rudolph and Hammen, 1999; Rudolph et al., 2000).

MRI acquisition. High-resolution anatomical MRI images were obtained using a 3-Tesla GE SIGNA (General Electric Medical Systems) scanner with a quadrature head RF coil. A three-dimensional, inversion recovery (IR) pulse sequence was used to generate T1-weighted images with 128 axial slices ( $1.2 \mathrm{~mm}$ thick) covering the whole brain. Before MRI scanning, participants were oriented to the MRI through the use of a mock-MRI simulator. During MRI acquisition, participants were instructed to stay as still as possible and were able to watch a movie of their choosing.

Imaging processing and template creation. T1-weighted image were reconstructed via Analysis of Functional NeuroImages (Cox, 1996), converted to ANALYZE format, and corrected for field inhomogenity via N3 (Sled et al., 1998). These images were then masked to include brain tissue and ventricular CSF. These masked images were next used to construct an optimal, population-specific template, using a diffeomorphic shape and intensity averaging technique (Avants and Gee, 2004; Avants et al., 2008). The MRI template was study-specific, constructed based on all subjects, containing children who had suffered maltreatment and children who had not suffered such adverse early stress. Template construction consisted of a multiresolution strategy (for this study, a four level Gaussian pyramid) as well as the similarity metric for the optimization, along with a maximum number of iterations. We used the region-based cross-correlation similarity metric, which is optimal in dealing with locally varying inhomogeneity in the appearance of images. The maximum number of iterations in the normalization was set to 200 , although convergence may occur before the maximum is reached. This processing yielded an unbiased average shape and appearance template, as well as the set of diffeomorphisms and inverse diffeomorphisms that map from template to each individual.

Symmetric diffeomorphic image normalization and tensor-based morphometry. After going through a 6-parameter rigid-body transformation, each individual brain was registered to our template using symmetric normalization (SyN). This algorithm allows for large deformations, but also constrains the deformations to be physically reasonable. The nonlinear transformations resulting from the SyN algorithm also provide deformation tensor fields, defined in the optimal template space, that describe the voxelwise shape change from the template to each subject's brain. Jacobian determinants of the deformation field indicate the fractional volume expansion and contraction at each voxel, quantifying the magnitude of regional volume alterations required to match the template. Before the statistical testing, this adjusted Jacobian map was subjected to a log transformation to make the distribution closer to the normal distribution (Avants and Gee, 2004). Jacobian determinants were then smoothed with a $4 \mathrm{~mm}$ full-width, half-maximum Gaussian filter.

Statistical analysis. Voxelwise group $t$ tests and whole brain regressions were next conducted in FMRISTAT (Worsley et al., 2002). Whole-brain volume and pubertal status were entered into a linear regression model as nuisance variables. An initial statistical threshold of $t_{(68)}=2.6503, p=$ 0.005 uncorrected, was used in examining any possible brain differences. Signal above this threshold was then corrected using Gaussian randomfield theory (Worsley et al., 2004) to limit type I error. Using this approach, regions needed to have a peak $t>2.6503$ and an extent $>284$ voxels, which was equivalent to $p \leq 0.05$ corrected.

In addition to examining group differences, voxelwise correlations were also conducted with scores from the LSI subscales. These analyses were only conducted within the abused samples, as significant group differences were detected on all of the LSI subscales. These correlations 
Table 1. Brain regions: physically abused < control

\begin{tabular}{|c|c|c|c|c|}
\hline Brain area & $\begin{array}{l}\text { Cluster size } \\
\text { (1 mm voxels) }\end{array}$ & $\begin{array}{l}p \text { value (corrected } \\
\text { via random field theory) }\end{array}$ & Peak within cluster & $\begin{array}{l}\text { Approximate coordinates } \\
\text { in MNI space }(x, y, z)\end{array}$ \\
\hline Right middle temporal lobe & 2129 & $p<0.001$ & 5.18 & $+54,-8,+18$ \\
\hline Right orbitofrontal cortex & 1925 & $p<0.001$ & 3.93 & $+34,+35,-8$ \\
\hline Right inferior temporal lobe/temporal pole & 1442 & $p<0.001$ & 4.5 & $+49,+7,-26$ \\
\hline Bilateral thalamus & 1354 & $p<0.001$ & 5.55 & $-4,-9,+5$ \\
\hline Right parietal lobe & 1108 & $p<0.001$ & 4.44 & $+51,-27,+25$ \\
\hline Right posterior parietal lobe & 894 & $p<0.001$ & 3.54 & $+30,-56,+38$ \\
\hline Right dorsolateral PFC & 634 & $p<0.001$ & 4.06 & $+16,+52,-42$ \\
\hline Right ventral-medial PFC & 626 & $p<0.001$ & 4.18 & $+10,+53,-22$ \\
\hline Right inferior temporal lobe/temporal pole & 576 & $p<0.001$ & 3.99 & $+33,+14,-38$ \\
\hline Left inferior temporal lobe/temporal pole & 555 & $p<0.001$ & 3.99 & $-36,+7,-36$ \\
\hline Left superior frontal lobe & 499 & $p=0.001$ & 4.22 & $-22,-1,+68$ \\
\hline Right frontal cortex & 442 & $p=0.002$ & 3.68 & $+59,+9,+32$ \\
\hline Left dorsolateral PFC & 407 & $p=0.004$ & 3.51 & $-16,+42,+45$ \\
\hline Left middle temporal gyrus & 368 & $p=0.009$ & 3.46 & $-63,-18,-19$ \\
\hline Left parietal cortex & 342 & $p=0.015$ & 3.94 & $-49,-31,+14$ \\
\hline Right superior temporal & 335 & $p=0.017$ & 3.26 & $+49,-28,+2$ \\
\hline Left vermis & 316 & $p=0.025$ & 3.61 & $-6,-67,-43$ \\
\hline Left postcentral & 286 & $p=0.045$ & 4.22 & $-59,-22,+46$ \\
\hline
\end{tabular}

Table 2. Brain regions: physically abused > control

\begin{tabular}{|c|c|c|c|c|}
\hline Brain area & $\begin{array}{l}\text { Cluster size } \\
\text { (1 mm voxels) }\end{array}$ & $\begin{array}{l}p \text { value (corrected } \\
\text { via random field theory) }\end{array}$ & Peak within cluster & $\begin{array}{l}\text { Approximate coordinates } \\
\text { in MNI space }(x, y, z)\end{array}$ \\
\hline Right occipital lobe & 2347 & $p<0.001$ & 4.93 & $+39,-82,+30$ \\
\hline Right cingulate & 586 & $p<0.001$ & 4.07 & $+4,-17,+51$ \\
\hline Left cerebellar white matter & 557 & $p<0.001$ & 5.25 & $-26,-38,-42$ \\
\hline Left frontal white matter & 479 & $p=0.001$ & 4.89 & $-17,+39,+28$ \\
\hline Left lateral cerebellum & 306 & $p=0.003$ & 3.83 & $-47,-48,-30$ \\
\hline Right cerebellum white matter & 296 & $p=0.037$ & 3.35 & $+26,-53,-36$ \\
\hline
\end{tabular}

Table 3. Additional results from logical AND conjunction analyses

\begin{tabular}{|c|c|c|c|}
\hline Brain area & Logical AND conjunction of & Cluster size (1 mm voxels) & $\begin{array}{l}\text { Approximate coordinates } \\
\text { in MNI space }(x, y, z)\end{array}$ \\
\hline Left parietal lobe & physically abused $<$ control group brain differences AND negative correlation LSI academic & 34 & $-58,-22,+44$ \\
\hline Right temporal lobe & physically abused < control group brain differences AND negative correlation LSI academic & 14 & $+51,-30,+1$ \\
\hline Right cingulate & physically abused $>$ control group brain differences AND positive correlation LSI academic & 7 & $+88,+107,+123$ \\
\hline Left prefrontal white matter & physically abused $>$ control group brain differences AND positive correlation LSI behavioral & 15 & $+104,+169,+99$ \\
\hline
\end{tabular}

$p$ value for all clusters, $p<0.000025$, uncorrected.

were then used in a logical AND conjunction analysis to identify the brain regions that were different between groups and also related to functioning for the physically abused sample in various domains as assessed by the LSI. This analytical approach has been used in a number of research studies (Nichols et al., 2006); such an approach is a straightforward way to find brain regions different between groups and also correlated with behavioral outcomes, with minimal statistics.

Group differences and correlation with the LSI were both thresholded at $p=0.005$, uncorrected (e.g., LSI correlation threshold $t_{(29)}=3.047$ ) and combined to see any overlapping regions. Brain group differences from the first step of our analyses at $p=0.05$ corrected were used to mask these logical AND conjunction analyses. In addition, a minimum clusterthreshold of 5 voxels or greater was set for all logical AND conjunctions to further minimize false-positives. Assuming independence of this test, these results are significant at $0.000025,\left(0.005^{\star} 0.005\right)$, uncorrected. The peak coordinates for all analyses were mapped to MNI space by registering the custom template to the MNI152 Average Template (Montreal Neurological Institute, Montreal, Canada) as presented in Tables 1, 2, and 3, to allow comparison from other studies.

\section{Results}

\section{Brain measurements}

As hypothesized, one of the largest differences between abused and nonabused children was in the right oFC, where those who had experienced physical abuse had smaller brain volumes (extent $=1.925 \mathrm{~cm}^{3} ; p<0.001$ corrected, peak $t$ in cluster $\left.=3.93\right)$. To ensure specificity of this result, this finding is corrected for individual differences in the overall sizes of children's brains (whole-brain volume) and each child's pubertal status. Tables 1 and 2 report other brain regions where victims of abuse differed from controls.

Compared with nonmaltreated control subjects, subjects who had been victims of physical abuse showed volume alterations in numerous areas throughout the brain. Table 1 details regions where significantly smaller volumes were found in children who had been victims of abuse. In brief, smaller volumes in physically abused subjects were noted in the right temporal, right frontal, and bilateral parietal lobes. Within the right temporal lobe, the greatest differences were noted in the middle and inferior portions, while in the frontal lobe, large changes were noted in the orbital frontal and dorsolateral-prefrontal cortices. Additionally, major alterations were noted bilaterally in the thalamus, with the greatest difference in the left thalamus. Table 2 reports the brain regions where significant larger volumes for physically abused children (compared with typically developing nonmaltreated children) were noted. Larger volumes for physically abused subjects were specifically found in the right posterior cingulate and in 


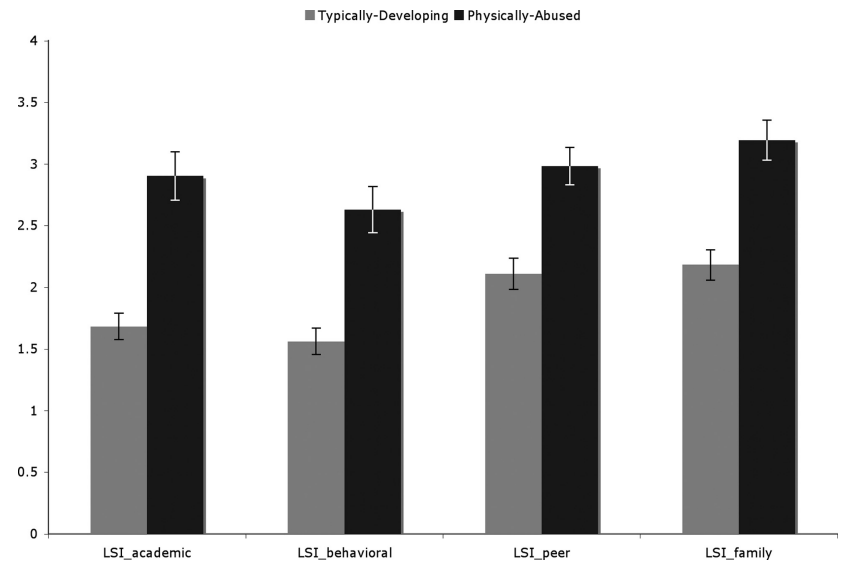

Figure 1. Results from LSI subscales for nonmaltreated control and physically abused adolescents. The figure shows that children who had been the victims of physical abuse reported more stressors in their academic, behavioral, peer, and family domains ( $p<0.001$ for each domain).

are regarding objective, yet contextually relevant indices of chronic stress.

\section{Relationships between oFC and social functioning}

To examine relationships between the oFC development and children's behavioral functioning, we used logical AND conjunction analyses to investigate whether a particular brain area that is different between groups is also the same area that is related to behavioral variables within the physically abused group alone (Friston et al., 1999). This approach is described in Materials and Methods, Statistical analysis. As hypothesized, smaller volumes in the right oFC observed in the physically abused children predicted problems in children's functioning (all regions, $p<0.000025$, uncorrected). The partial correlation between oFC volume and the family stress scale was $r=-0.516, p=0.004$. Greater difficulties in the child-parent relationship, such as more frequent arguments and less emotional support, were associated with smaller oFC volumes (Fig. 2a). The partial correlation between oFC volume and children's experience of academic problems/school stress was $r=-0.504, p=0.005$ (Fig. 2b).

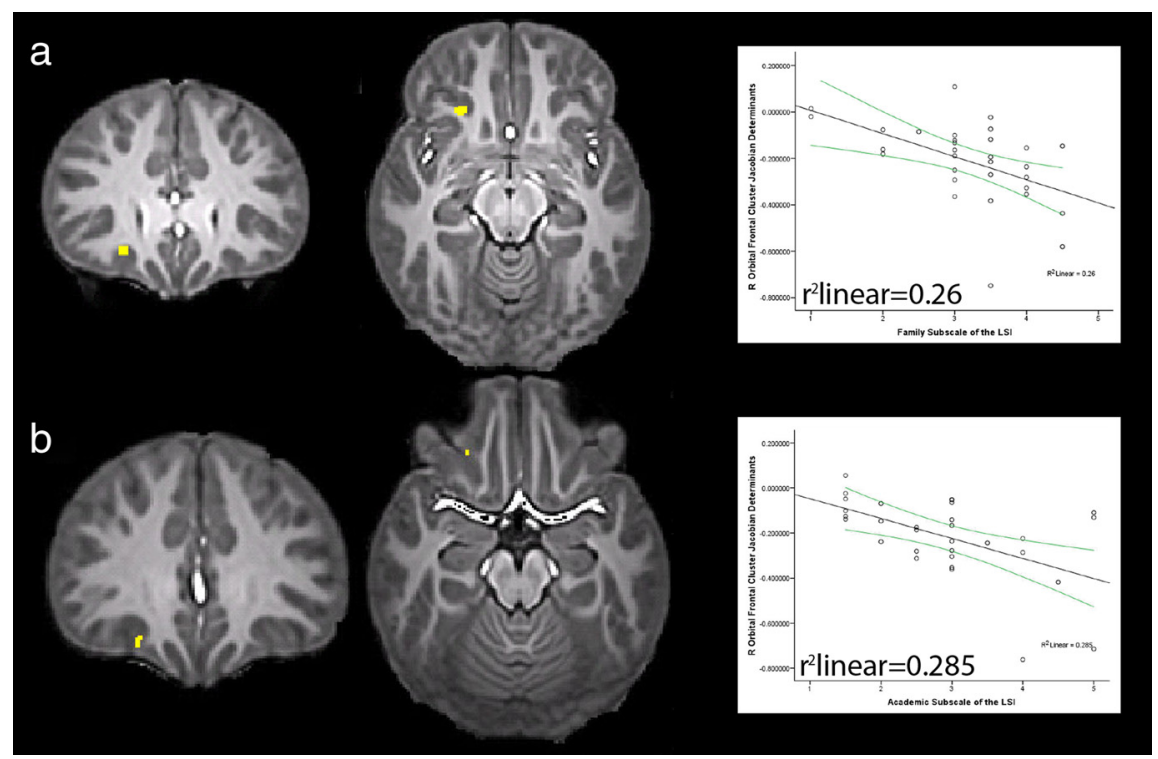

Figure 2. Logical AND conjunction analyses for the oFC. $\boldsymbol{a}$, The oFC cluster was smaller in physically abused children compared with controls, and there is a significant negative relationship between oFC development and the occurrence of family stress. $\boldsymbol{b}$, The oFC cluster was smaller in physically abused subjects compared with controls and shows a significant negative relationship between oFC development and school-related stress. For both clusters, smaller volumes (mean Jacobian determinants shown on the ordinate of each scatterplot) were related to increasing difficultly in social functioning; both clusters, $p<0.000025$, uncorrected). These analyses are corrected for individual differences in whole brain volume and pubertal status.

white matter in the cerebellum and prefrontal cortex. Additionally, major alterations were noted in occipital lobe. All of these alterations take into account variation in whole-brain volume and pubertal status.

\section{Social functioning}

Children who had been the victims of physical abuse reported more stressors in their academic (typically developing: $1.6829 \pm$ 0.6869; physically abused: $2.9032 \pm 1.075634346 ; F=34.338$, $p<0.001$ ), behavioral (typically developing: $1.5609 \pm 0.6818$; physically abused: $2.6290 \pm 1.0244 ; F=28.146, p<0.001$ ), peer (typically developing: $2.1097 \pm 0.8102$; physically abused: $2.9839 \pm 0.8214 ; F=20.305, p<0.001$ ), and family (typically developing: $2.1829 \pm 0.7886$; physically abused: $3.1935 \pm 0.8914$; $F=25.907, p<0.001$ ) domains (Fig. 1). Although the LSI probes both subjective and objective aspects of stress, these differences Greater academic difficulties, such as failure of 3 or more subjects in 1 year and full-time placement in a learning disabilities class, were associated with smaller oFC volumes. Both of these correlations controlled for variation between individual children in whole-brain volume and pubertal status.

\section{Relationships between} neuropsychological functioning and brain measurements CANTAB data were only available for 41 total subjects (14 physically abused; 27 nonmaltreated controls). To test this subset of participants did not differ from the full sample in any systematic way, we dummy coded CANTAB data availability and entered this into a linear regression as an independent variable to test for relationship between other variables of interest, which were entered as dependent variables. CANTAB data availability was not related to SES, as indexed by maternal education, $(p=0.170)$ or OFC volume $(p=0.864)$. To test whether neuropsychological functioning was related to the differences in orbitofrontal volumes found across group, we constructed a factor measure of neuropsychological performance, which included the Intra/Extra Dimensional Set-Shift, Paired Associates Learning, Pattern Recognition Memory, Stockings of Cambridge, and Spatial Working Memory CANTAB subtests. This factor measure was computed for each subject by averaging the individual $z$-scores output from each test and then was entered into a linear regression equation. No significant association between orbitofrontal cortex and neuropsychological functioning was found $(p=0.72)$ and maltreatment group remained significantly related to orbitofrontal volume $(p<0.001)$.

\section{Relationships between socioeconomic status and} brain measurements

To test whether the differences seen in orbitofrontal volumes across group were related to socioeconomic status (SES), years of maternal 
education, a commonly used proxy of SES, was entered into a linear regression, with orbitofrontal volumes as a dependent variable and group and years of maternal education as the independent variables. No significant association emerged between orbitofrontal cortex and SES ( $p=0.90)$, and maltreatment group remained significant related to Orbitofrontal Volume after controlling for SES ( $p=$ $0.034)$. We repeated these regressions using every index of socioeconomic status available to us, including Median Household Income and Median Family Income from U.S. Census Tract data; in all cases the relationship between maltreatment group and orbitofrontal volume remained significant while the relationship between SES variables and orbitofrontal volume was not significant.

\section{Relationships between other brain structures and life stress}

Regions in addition to the oFC were different between groups and related to behavioral functioning (Table 3). For academic functioning, the smaller volumes in left parietal lobe and right temporal lobe seen initially in physically abused children displayed a significant negative relationship with functioning, with smaller volumes seen with increasing problems in this domain. Larger volumes for the physically abused sample in the Right Cingulate displayed a significant positive relationship with impaired functioning in the academic domain, with larger volumes seen with greater functional impairments. In the Behavioral domain, larger white matter volumes in left PFC were positively correlated with functioning in the broad behavioral domain as assessed by the Life-Stress Interview with greater volumes in the brain region related to increasing problems.

\section{Discussion}

Our findings indicate that oFC volumes are smaller in children who have suffered early aberrant parental care in the form of physical abuse, and that these volumetric alterations are associated with difficulties children experience in various aspects of their social lives. The oFC is a key component of a circuit that aids in adaptation to changing environmental contingencies and plays an important role in the control of emotion and motivational states. The structural alterations that we observed in abused childrens' oFC may lead to functional difficulties for these children, compromising their abilities to navigate in and adaptively regulate to changing social contexts.

The data reported here are consistent with studies of rodents and primates that indicate that early stress affects development of the PFC (Arnsten et al., 2009). Moreover, previous research examining neurological-damaged patients with oFC lesions indicate that damage to this brain region has been linked with deficits in emotion, mood, and social regulation (Damasio, 1994). Although previous neuroimaging investigations have noted structural brain differences among maltreated children (De Bellis et al., 1999, 2002; Richert et al., 2006; Carrion et al., 2009), extant studies have not found robust connections between brain and behavior. This may be due to the fact that previous studies have tested samples of children who experienced maltreatment but who also had concurrent psychiatric conditions such as posttraumatic stress disorder (PTSD). In the physically abused group in this study, two children met DSM IV criteria for conduct disorder, two others met criteria for an eating disorder, and one child had experienced a major depressive episode previously. In addition, children who suffered physical abuse reported more problems in all the domains assayed by the LSI (Fig. 1, shown). As recently reviewed by Gilbert et al. (2009), child maltreatment such as physical abuse, has long-lasting effects on many diverse facets of mental health, drug and alcohol misuse, risky sexual behavior, obesity, and criminal behavior. Therefore, we did not wish to limit the study to individuals with one discrete disorder.

Another challenge faced by previous investigations is that children's brains are difficult to measure. Methodologically, our study design used a rigorous analytic approach, TBM, with information generated from a powerful registration algorithm, SyN (Avants and Gee, 2004). In a recent comparison of 14 nonlinear registration algorithms, SyN emerged as one of the best available warping algorithms (Klein et al., 2009). This approach represents an important advance because other analytic approaches for structural imaging data, such as parcellation or voxel-based morphometry, may especially limit studies of children's brain development. Parcellation divides the brain into large zones, but in doing so, aggregates functionally divergent brain regions together, each of which may be differentially involved in behavioral outcomes (Gazzaniga et al., 2008). Voxelbased morphometry (VBM), a commonly used approach, may be less reliable with pediatric populations because of the use of adult anatomical templates and spatial normalization parameters (Bookstein, 2001; Wilke et al., 2003). Special care, such as creation of a study-specific template and use of appropriate a priori segments, should be taken with VBM processing to address these issues (Peper et al., 2009). The approach we employ here, TBM, minimizes these sources of variability (e.g., brain tissue segmentation), uses a study-specific anatomical template and yields high sensitivity at the voxel level.

Of important note, the structural imaging study presented here has aspects of its design that potentially limit the interpretation of results. Neurobiological research with high-risk samples, such as the maltreated children, is challenging due to potential third variables that could affect brain development or other biological variables. For example, a proportion of the brain differences reported could be due genetic effects. Behavioral genetics research in the area of child maltreatment however underscores the limited effects of genetic factors; instead this body of research underscores a critical role for early postnatal experience in the outcomes of abused children (Pollak, 2008; Kim-Cohen and Gold, 2009). In addition, we did not have access to the health records of parents or children, making it difficult to rule out the effects of exposure to teratogens. Importantly, prenatal alcohol exposure and broad neuropsychological differences were controlled for in this sample, supporting our interpretation of the data and specificity of the effect. Also, we did not have information regarding the time course of maltreatment in this sample. Individual differences exist in severity and chronicity of maltreatment, along with access to psychotherapeutic services after an abuse episode. This information would aid in understanding the effects of early adversity on brain-behavior relationships, as the characteristics of maltreatment has been linked with differential psychological outcomes (Manly et al., 1994).

Regions in addition to the oFC were different between groups and related to behavioral functioning (Table 3). These findings may have important implications for brain development and understanding the neurobiological correlates of maltreatment. Additional research is needed to understand how these other regions may be related to the trajectory of risk conveyed by physical abuse.

By specifically linking child maltreatment to focal neuroanatomical alterations and those alterations to behavioral changes, our results suggest one potential mechanism, structural changes in the oFC, through which child maltreatment may put individuals at greater risk for a variety of behavioral problems throughout their lives. This brain region supports the monitoring of 
behavioral outcomes (Kringelbach and Rolls, 2004) and regulation of affective states (Lévesque et al., 2004; Ohira et al., 2006; Urry et al., 2006)—domains of behavior frequently affected in children who have been physically abused (Brown, 2003). Importantly, early dysfunction of the oFC may not become apparent until adolescence or early adulthood, when this brain region becomes fully functional (Bachevalier and Loveland, 2006). This may account for why some behavioral problems in previously maltreated individuals do not manifest in early childhood. Further study is needed to more fully understand the brain-behavior effects of early postnatal social experience. One limitation of our design is that the results are based upon one time point, therefore we cannot discern whether oFC development is slowed or significantly delayed. For this reason, we refer to "differences" or "alterations" in the describing our findings. As a next step, functional neuroimaging, in conjunction with anatomical measurement, may be used to examine how structural variation in the oFC might be related to variations in functional connectivity between the oFC and other brain regions that convey behavioral risk from child abuse. Arnsten (2009) theorized that chronic stress, such as maltreatment, may weaken the regulatory abilities of structures that provide negative feedback on the stress response (such as the PFC), but also bolster the structures that promote the stress response, such as the amygdala. To date, there is little known about molecular stress-signaling mechanisms likely to be related to morphological alterations of the PFC; one candidate might be alterations of catecholamine pathways and increasing noradrenergic innervation of the PFC leading to higher levels of phosphatidylinositol-protein kinase $\mathrm{C}$ and cyclic adenosine monophosphate signaling (Arnsten, 2009). Preliminary research (De Bellis et al., 1994) as found alterations in catecholamine excretion in a small sample of abused girls, providing initial support for alterations in this stress-signaling cascade. Future studies could combine these types of physiological metrics with structural neuroimaging using a design similar to the one used here.

Overall, children who were victims of physical abuse had alterations in oFC and these alterations were related to functioning in different domains of behavior. This finding suggests a potential neurobiological mechanism through which early adverse experiences constitute risks for children's cognitive and emotional development.

\section{References}

Arnsten AF (2009) Stress signalling pathways that impair prefrontal cortex structure and function. Nat Rev Neurosci 10:410-422.

Astley SJ, Stachowiak J, Clarren SK, Clausen C (2002) Application of the fetal alcohol syndrome facial photographic screening tool in a foster care population. J Pediatr 141:712-717.

Avants B, Gee JC (2004) Geodesic estimation for large deformation anatomical shape averaging and interpolation. Neuroimage 23:S139-S150.

Avants BB, Epstein CL, Grossman M, Gee JC (2008) Symmetric diffeomorphic image registration with cross-correlation: evaluating automated labeling of elderly and neurodegenerative brain. Med Image Anal 12:26-41.

Bachevalier J, Loveland KA (2006) The orbitofrontal-amygdala circuit and self-regulation of social-emotional behavior in autism. Neurosci Biobehav Rev 30:97-117.

Bookstein FL (2001) "Voxel-based morphometry" should not be used with imperfectly registered images. Neuroimage 14:1454-1462.

Braun K, Lange E, Metzger M, Poeggel G (2000) Maternal separation followed by early social deprivation affects the development of monoaminergic fiber systems in the medial prefrontal cortex of Octodon degus. Neuroscience 95:309-318.

Brown EJ (2003) Child physical abuse: risk for psychopathology and efficacy of interventions. Curr Psychiatry Rep 5:87-94.
Carrion VG, Weems CF, Watson C, Eliez S, Menon V, Reiss AL (2009) Converging evidence for abnormalities of the prefrontal cortex and evaluation of midsagittal structures in pediatric posttraumatic stress disorder: an MRI study. Psychiatry Res 172:226-234.

Chung MK, Worsley KJ, Paus T, Cherif C, Collins DL, Giedd JN, Rapoport JL, Evans AC (2001) A unified statistical approach to deformation-based morphometry. Neuroimage 14:595-606.

Cicchetti D, Carlson V (1989) Child maltreatment: theory and research on the causes and consequences of child abuse and neglect. New York: Cambridge UP.

Cox RW (1996) AFNI: software for analysis and visualization of functional magnetic resonance neuroimages. Comp Biomed Res 29:162-173.

Damasio AR (1994) Descartes' error: emotion, reason, and the human brain. New York: GP Putnam.

De Bellis MD, Lefter L, Trickett PK, Putnam FW Jr (1994) Urinary catecholamine excretion in sexually abused girls. J Am Acad Child Adolesc Psychiatry 33:320-327.

De Bellis MD, Keshavan MS, Clark DB, Casey BJ, Giedd JN, Boring AM, Frustaci K, Ryan ND (1999) A.E. Bennett Research Award. Developmental traumatology part II: brain development. Biol Psychiatry 45: 1271-1284.

De Bellis MD, Keshavan MS, Shifflett H, Iyengar S, Beers SR, Hall J, Moritz G (2002) Brain structures in pediatric maltreatment-related posttraumatic stress disorder: a sociodemographically matched study. Biol Psychiatry 52:1066-1078.

Ellis BJ, Garber J (2000) Psychosocial antecedents of variation in girls' pubertal timing: maternal depression, stepfather presence, and marital and family stress. Child Dev 71:485-501.

Friston KJ, Holmes AP, Price CJ, Büchel C, Worsley KJ (1999) Multisubject fMRI studies and conjunction analyses. Neuroimage 10:385-396.

Gazzaniga MS, Ivry R, Mangun GR (2008) Cognitive neuroscience: the biology of the mind, Ed 3. New York: WW Norton.

Genentech (1997) Assessment of pubertal stages, G70577-R1\#LF0050. San Francisco: Genentech.

Gilbert R, Widom CS, Browne K, Fergusson D, Webb E, Janson S (2009) Burden and consequences of child maltreatment in high-income countries. Lancet 373:68-81.

Helmeke C, Seidel K, Poeggel G, Bredy TW, Abraham A, Braun K (2009) Paternal deprivation during infancy results in dendrite- and time-specific changes of dendritic development and spine formation in the orbitofrontal cortex of the biparental rodent, Octodon degus. Neuroscience 163:790-798

Kim-Cohen J, Gold AL (2009) Measured gene-environment interactions and mechanisms promoting resilient development. Curr Dir Psychol Sci $18: 138-142$.

Klein A, Andersson J, Ardekani BA, Ashburner J, Avants B, Chiang MC, Christensen GE, Collins DL, Gee J, Hellier P, Song JH, Jenkinson M, Lepage C, Rueckert D, Thompson P, Vercauteren T, Woods RP, Mann JJ, Parsey RV (2009) Evaluation of 14 nonlinear deformation algorithms applied to human brain MRI registration. Neuroimage 46:786-802.

Kringelbach ML, Rolls ET (2004) The functional neuroanatomy of the human orbitofrontal cortex: evidence from neuroimaging and neuropsychology. Prog Neurobiol 72:341-372.

Lévesque J, Joanette Y, Mensour B, Beaudoin G, Leroux JM, Bourgouin P, Beauregard M (2004) Neural basis of emotional self-regulation in childhood. Neuroscience 129:361-369.

Manly JT, Cicchetti D, Barnetta D (1994) The impact of subtype, frequency, chronicity, and severity of child maltreatment on social competence and behavior problems. Dev Psychopathol 6:121-143.

Marshall WA, Tanner JM (1969) Variations in puberty changes in girls. Arch Dis Child 44:291-303.

Marshall WA, Tanner JM (1970) Variations in the puberty changes in boys. Arch Dis Child 45:13-23.

Nichols EA, Kao YC, Verfaellie M, Gabrieli JD (2006) Working memory and long-term memory for faces: Evidence from fMRI and global amnesia for involvement of the medial temporal lobes. Hippocampus 16:604-616.

Ohira H, Nomura M, Ichikawa N, Isowa T, Iidaka T, Sato A, Fukuyama S, Nakajima T, Yamada J (2006) Association of neural and physiological responses during voluntary emotion suppression. Neuroimage 29:721-733.

Paulus MP (2007) Decision-making dysfunctions in psychiatry-altered homeostatic processing? Science 318:602-606. 
Peper IS, Schnack HG, Brouwer RM, Van Baal GC, Pjetri E, Székely E, van Leeuwen M, van den Berg SM, Collins DL, Evans AC, Boomsma DI, Kahn RS, Hulshoff Pol HE (2009) Heritability of regional and global brain structure at the onset of puberty: a magnetic resonance imaging study in 9-year-old twin pairs. Hum Brain Mapp 30:2184-2196.

Pollak SD (2008) Mechanisms linking early experience and the emergence of emotions, illustrations from the study of maltreated children. Curr Dir Psychol Sci 17:370-375.

Reich W (2000) Diagnostic interview for children and adolescents. J Am Acad Child Adolesc Psychiatry 39:59-66.

Rempel-Clower NL (2007) Role of orbitofrontal cortex connections in emotion. Ann N Y Acad Sci 1121:72-86.

Richert KA, Carrion VG, Karchemskiy A, Reiss AL (2006) Regional differences of the prefrontal cortex in pediatric PTSD: an MRI study. Depress Anxiety 23:17-25.

Rudolph KD, Hammen C (1999) Age and gender as determinants of stress exposure, generation, and reactions in youngsters: a transactional perspective. Child Dev 70:660-677.

Rudolph KD, Hammen C, Burge D, Lindberg N, Herzberg D, Daley SE (2000) Toward an interpersonal life-stress model of depression: the developmental context of stress generation. Dev Psychopathol 12:215-234.

Sánchez MM, Hearn EF, Do D, Rilling JK, Herndon JG (1998) Differential rearing affects corpus callosum size and cognitive function of rhesus monkeys. Brain Res 812:38-49.
Shirtcliff EA, Dahl RE, Pollak SD (2009) Pubertal development: correspondence between hormonal and physical development. Child Dev 80:327-337.

Sled JG, Zijdenbos AP, Evans AC (1998) A nonparametric method for automatic correction of intensity nonuniformity in MRI data. IEEE Trans Med Imag 17:87-97.

Straus MA, Hamby SL, Finkelhor D, Moore DW, Runyan D (1998) Identification of child maltreatment with the Parent-Child Conflict Tactics Scales: development and psychometric data for a national sample of American parents. Child Abuse Negl 22:249-270.

Toga AW, Thompson PM, Sowell ER (2006) Mapping brain maturation. Trends Neurosci 29:148-159.

Urry HL, van Reekum CM, Johnstone T, Kalin NH, Thurow ME, Schaefer HS, Jackson CA, Frye CJ, Greischar LL, Alexander AL, Davidson RJ (2006) Amygdala and ventromedial prefrontal cortex are inversely coupled during regulation of negative affect and predict the diurnal pattern of cortisol secretion among older adults. J Neurosci 26:4415-4425.

Wilke M, Schmithorst VJ, Holland SK (2003) Normative pediatric brain data for spatial normalization and segmentation differs from standard adult data. Magn Reson Med 50:749-757.

Worsley KJ, Liao CH, Aston J, Petre V, Duncan GH, Morales F, Evans AC (2002) A general statistical analysis for fMRI data. Neuroimage 15:1-15.

Worsley KJ, Taylor JE, Tomaiuolo F, Lerch J (2004) Unified univariate and multivariate random field theory. Neuroimage 23:S189-195. 